\title{
Estado nutricional de indígenas Kaingang e Guarani no estado do Paraná, Brasil
}

\author{
The nutritional status of Kaingang and Guarani indigenous peoples \\ in the State of Paraná, Brazil
}

Juliana Dias Boaretto ${ }^{1}$

Carlos Alexandre Molena-Fernandes ${ }^{1}$

Giuliano Gomes de Assis Pimentel ${ }^{2}$

${ }^{1}$ Departamento de Educação Física, Centro de Ciências da Saúde, Universidade Estadual do Paraná. Av. Gabriel Esperidião s/n, Jardim Morumbi. 87703-000 Paranavaí PR Brasil. julivictor@yahoo.com.br ${ }^{2}$ Departamento de Educação Física, Centro de Ciências Biológicas, Universidade Estadual de Maringá.

\begin{abstract}
This study arose from the need to comprehend epidemiological aspects to establish a policy for physical activity for indigenous peoples. Although infectious diseases are still the main causes of suffering in these ethnic groups, chronic diseases have emerged due to the process of epidemiological/nutritional change in indigenous peoples subject to the policy of life on reservations. The scope of this study was to evaluate the nutritional status of indigenous peoples belonging to two ethnic groups in the State of Paraná. Anthropometric data were collected on 178 adults belonging to the Kaingang $(n=117)$ and Guarani $(n=61)$ indigenous ethnic groups. The prevalence of being overweight in Guarani and Kaingang adults was $32.3 \%$ and $41 \%$, respectively, detecting a prevalence of obesity in the order of $3.2 \%$ among the Guarani indigenous people and $12.8 \%$ among the Kaingang ethnic group. Anthropometric changes observed among the Guarani and Kaingang indigenous peoples of Paraná are of increasing concern according to some studies. Thus, the results of this study reinforce the need for integrated actions such as nutritional guidance and physical activity during leisure time for the promotion of the health of these populations.
\end{abstract}

Key words Nutritional status, Obesity, Physical exercise, Health of indigenous peoples
Resumo Este estudo surgiu da necessidade em conhecer aspectos epidemiológicos que orientassem uma política de atividade física junto às etnias indígenas. Embora as doenças infecciosas ainda sejam as principais causadoras de padecimento nessas populações, as doenças crônicas já se tornaram evidentes graças ao processo de mudança epidemiológica/nutricional nos grupos indígenas sujeitos à política de reservas. O objetivo deste estudo foi avaliar o estado nutricional de indígenas de duas etnias do Paraná. Dados antropométricos foram coletados em 178 indígenas adultos pertencentes às etnias Kaingang $(n=117)$ e Guarani $(n$ =61). A prevalência de sobrepeso em Guarani e Kaingang adultos foi de 32,3 e 41,0\%, respectivamente, detectando-se prevalência de obesidade da ordem de 3,2\% entre os indígenas Guarani e 12,8\% para os da etnia Kaingang. As alterações antropométricas observadas entre os indígenas Guarani e Kaingang do Paraná são cada vez mais preocupantes segundo algumas pesquisas. Sendo assim, os resultados deste estudo reforçam a necessidade de ações integradas a exemplo da orientação nutricional e à atividade física no lazer em prol da promoção da saúde dessas populações.

Palavras-chave Estado nutricional, Obesidade, Exercício físico, Saúde de Populações Indígenas 


\section{Introdução}

Atualmente no Brasil, segundo dados do Censo 2010 do Instituto Brasileiro de Geografia e Estatística, a população indígena aproxima-se de 900 mil pessoas ${ }^{1}$. Destes, 324.834 vivem em cidades e $572.083 \mathrm{em}$ áreas rurais, o que equivale a 0,47\% da população total brasileira. A maior parte dessa população distribui-se por milhares de aldeias, situadas no interior de 693 Terras Indígenas, de norte a sul do território nacional ${ }^{1}$. Em ambiente menos silvícola, hábitos alimentares e atividade físico-esportiva autóctones foram drasticamente alterados, afetando o perfil epidemiológico desses povos.

O presente estudo abordou a ocorrência da obesidade nas duas principais etnias presentes no Paraná, os Guarani e os Kaingang, que estão entre as três mais populosas no Brasil. O povo Guarani constitui uma das populações indígenas de maior presença territorial no continente sul-americano. Segundo o ISA $^{2}$, este povo vive em regiões no Brasil e em alguns países como Argentina, Bolívia e Paraguai e se diferenciam em diversos grupos, semelhantes entre si nos aspectos fundamentais de sua cultura e organizações sociopolíticas, bem como diferentes no modo de falar a língua Guarani, de praticar sua religião e distintos no que diz respeito às tecnologias que aplicam na relação com o meio ambiente.

Estudos de Hennerich ${ }^{3}$ ressaltam que a etnia Kaingang representa a terceira em população no Brasil, falam a língua do tronco linguístico Jê e encontram-se espalhados em quatro estados brasileiros sendo eles: São Paulo, Paraná, Santa Catarina e Rio Grande do Sul. Complementam Mota ${ }^{4}$ e o ISA ${ }^{2}$ que os Kaingang vivem em mais de 30 Terras Indígenas e representam uma pequena parcela de seus territórios tradicionais. Por estarem distribuídos em quatro estados, a situação não é homogênea. Em todos os casos, contudo, sua estrutura social e princípios cosmológicos continuam vigorando, atualizados frente às diferentes conjunturas históricas.

As duas etnias já citadas, somadas à Xetá, representam os três povos pertencentes ao estado do Paraná. De acordo com estudos de Boaretto 5 , ao realizar pesquisas em Terras Indígenas paranaenses, foram mapeadas 30 terras (terras demarcadas e não demarcadas), sendo 17 pertencentes a etnia Guarani, 8 à Kaingang, 2 às etnias Guarani e Kaingang, 2 com as etnias Guarani, Kaingang e Xetá e 1 terra desabitada.

Ao pensarmos a relação que os povos indígenas possuem com a saúde, é importante conside- rar a especificidade cultural de cada grupo. Não existe, a rigor, um indígena genérico, mas como Mota e Assis ${ }^{6}$ apontam, nas comunidades indígenas cada grupo desenvolve seu linguajar, pratica religião, apresenta sistema de parentesco próprio ocupando territórios específicos, vivendo a partir de normas morais e políticas próprias, o que nem sempre lhes confere as mesmas representações de corpo e práticas sociais de promoção da saúde e cura.

Entretanto, o contato com a cultura não indígena resultou em alguns efeitos homogeneizantes no plano sociocultural, especialmente no tocante à restrição espacial, infringindo uma estrutura sedentária em etnias historicamente nômades. Em acréscimo, não obstante as resistências autóctones, ocorreram mudanças em relação aos hábitos alimentares e atividade física, os quais atuam diretamente sobre o estado nutricional dessa população. Destarte, a saúde indígena é um objeto preocupante, devido às aceleradas mudanças nos perfis epidemiológicos deste grupo. Se as doenças infecciosas ainda continuam a ocupar um papel dominante, por outro lado se percebe crescimento na ocorrência de doenças crônicas, dentre estas aquelas associadas à obesidade ${ }^{7}$.

Desta forma, Taddei et al. ${ }^{8}$ enfatizam que as últimas duas décadas do século XX foram marcadas por várias modificações no perfil de saúde humana, apresentando um período de mudanças na estrutura da composição corporal e na dieta de indivíduos, associadas a fatores diretamente vinculados à atividade física e ao estilo de vida.

Logo, entre os povos indígenas, de acordo com Ribas et al. ${ }^{9}$, essas modificações afetam negativamente também sua alimentação, representada pelo empobrecimento das práticas alimentares, com a diminuição do consumo dos alimentos tradicionais. $\mathrm{Na}$ realidade indígena, esse empobrecimento da diversidade é um risco à segurança alimentar.

Diante deste cenário, começam a surgir estudos que busquem o entendimento sobre alguns aspectos existentes que, ao passar do tempo, vêm se modificando neste grupo social, como: saúde, modo de vida, alimentação, nutrição, atividade física, cultura, arte, educação, entre outros ${ }^{10}$. Mais especificamente, necessitávamos conhecer fatores relativos a condições de vida e saúde das diversas etnias indígenas que orientassem uma política de atividade física no tempo livre das suas populações, principalmente no Paraná, onde estudos epidemiológicos nesses povos são escassos. Nesta perspectiva, o presente estudo objetivou avaliar e comparar o estado nutricional de indígenas adul- 
tos, das etnias Kaingang e Guarani presentes no estado do Paraná.

\section{Métodos}

O presente estudo caracteriza-se como descritivo transversal e a coleta de dados foi realizada na cidade de Maringá, Paraná, com 178 indígenas adultos, pertencentes a etnia Kaingang $(\mathrm{n}=117)$ e Guarani ( $n=61)$, que participaram ou presenciaram o II Torneio de Futebol Indígena promovido pelo GEL/UEM (Grupo de Estudos do Lazer da Universidade Estadual de Maringá). Do total da amostra, 62 pessoas eram do sexo feminino e 116 do masculino, com idade entre 18 e 45 anos.

Inicialmente foi feito contato com os indígenas para informá-los sobre o objetivo do estudo e os procedimentos metodológicos. Na sequência, os sujeitos que concordaram em participar assinaram um termo de consentimento livre e esclarecido. O estudo atende à norma 196/96 ${ }^{11}$ do Conselho Nacional de Saúde, Ministério da Saúde, que rege sobre pesquisas com seres humanos, incluindo os cuidados relativos a povos indígenas, sendo aprovado pelo Comitê Permanente de Ética em Pesquisa Envolvendo Seres Humanos da Universidade Estadual de Maringá.

Os indicadores antropométricos mensurados foram peso e estatura e os dados foram avaliados pelo cálculo do IMC (índice de massa corporal), por meio da razão entre massa corporal $(\mathrm{kg})$ versus estatura $(\mathrm{m})$ elevada ao quadrado $(\mathrm{kg} /$ $\mathrm{m}^{2}$ ). Os pontos de corte utilizados foram os recomendados pela Organização Mundial de Saúde (1995), a saber: valores $<18,5$ igual a baixo peso, valores $\geq 18,5$ e $\leq 24,9$ igual a eutrofia, valores $\geq 25$ e $\leq 29,9$ igual a sobrepeso, valores $\geq 30$ e $\leq$ 34,9 igual a obesidade leve, valores $\geq 35$ e $\leq 39,9$ igual a obesidade moderada, e valores $\geq 40$ igual a obesidade grave. Para a verificação das medidas foi utilizada balança microeletrônica (marca Plena ${ }^{\circledR}$ ) com capacidade para $150 \mathrm{~kg}$ e estadiômetro adulto marca Exata ${ }^{\circledR}$ com resolução de $1 \mathrm{~mm}$.

Os dados do presente estudo foram tabulados e analisados no programa Statistic 11.0. A análise estatística utilizada foi descritiva, na qual foram calculados distribuição de frequência e medidas de tendência central (médias e desvios-padrão). Foi utilizado o teste Qui-Quadrado para comparar as prevalências de sobrepeso e obesidade entre as duas comunidades estudadas e o teste ' $\mathrm{t}$ ' de Student para comparação de médias de idade, utilizando como critério de significância $\mathrm{p}<$ 0,05 .

\section{Resultados e discussão}

Em nenhuma das duas etnias foram observadas diferenças significativas quanto às médias de idade entre os sexos. Também não houve diferenças significativas nas médias de idades quando comparados os dois grupos, demonstrando, desta forma, uma amostra homogênea em relação à faixa etária.

Quanto ao estado nutricional, as prevalências de sobrepeso e obesidade foram significativamente superiores entre os Kaingang, quando comparados aos Guaranis. O perfil do estado nutricional que emerge deste estudo apresenta semelhanças com o atual cenário da transição nutricional visto para a população brasileira. Analisando os valores de toda a amostra, pode ser observada uma quantidade preocupante de indígenas classificados acima do peso, pois somando os percentuais de sujeitos com sobrepeso e obesidade, verificou-se que quase metade dos avaliados $(47,9 \%)$ encontra-se com excesso de peso corporal (Tabela 1). Considerando que são indivíduos relativamente jovens, estes dados são ainda mais preocupantes, uma vez que está bem evidenciado na literatura que o excesso de peso em médio e longo prazo pode desencadear uma série de distúrbios orgânicos (Tabela 1).

Na população de adultos estudados Guarani e Kaingang, foram observados elevados percentuais de sobrepeso e obesidade, principalmente entre as mulheres (Tabelas 2 e 3 ). Ao comparar os dados deste estudo com outras comunidades, podem ser observados percentuais similares para sobrepeso, como, por exemplo, o registrado por Leite ${ }^{12}$, que encontrou $49,2 \%$ de sobrepeso ao avaliar o IMC de 63 mulheres adultas Xavante. Em estudos de Capelli e Koifman ${ }^{13}$, também foram observadas prevalências semelhantes de sobrepeso e obesidade em adultos indígenas Parkatêjê.

Dos 62 Guaranis avaliados, observou-se que mais de um terço $(35,5 \%)$ apresentou excesso de

Tabela 1. Estado Nutricional de Guarani e Kaingang adultos do Paraná.

\begin{tabular}{|c|c|c|c|c|c|c|}
\hline & \multicolumn{2}{|c|}{ Total $(n=179)$} & \multicolumn{2}{|c|}{ Guarani $(n=62)$} & \multicolumn{2}{|c|}{ Kaingang $(n=117)$} \\
\hline & f & $(\%)$ & f & $(\%)$ & f & $(\%)$ \\
\hline Baixo peso & 04 & $(2,2)$ & 03 & $(4,8)$ & 01 & $(0,9)$ \\
\hline Eutrófico & 90 & $(50,3)$ & 37 & $(59,7)$ & 53 & $(45,3)$ \\
\hline Sobrepeso & 68 & $(38,0)$ & 20 & $(32,3)$ & 48 & $(41,0)^{*}$ \\
\hline Obesidade & 17 & $(9,5)$ & 02 & $(3,2)$ & 15 & $(12,8)$ \\
\hline
\end{tabular}

"diferença significativa entre Guarani e Kaingang para p $<0,05$. $f=$ frequência. 
peso corporal, sendo que a prevalência de sobrepeso foi superior entre os homens e de obesidade superior entre as mulheres (Tabela 2).

Os dados revelam diferenças importantes entre os sexos quanto ao estado nutricional dos Kaingang (Tabela 3). Foi observada uma prevalência de sobrepeso de $45,9 \%$ em mulheres e $38,75 \%$ em homens, e a de obesidade de 21,7\% e $8,75 \%$, respectivamente. Estes percentuais indicam que mais da metade da população feminina $(67,6 \%)$ mostrou excesso de massa corporal total, representando uma prevalência de excesso de peso $20 \%$ superior à identificada entre os homens (Tabela 3). Corroborando com os achados deste estudo, Tavares et al. ${ }^{14}$, em pesquisa realizada com adultos indígenas de Suruí, Rondônia, verificaram a necessidade de elaboração de estratégias para prevenir e controlar os fatores de risco para doenças e agravos não transmissíveis, particularmente no grupo feminino.

Entretanto, alguns estudos apresentam dados que diferem dos resultados da presente pesquisa. Salvo et al. ${ }^{15}$, por exemplo, identificaram frequência maior de indígenas Suyá homens com excesso de peso quando comparado com as mulheres. Por outro lado, Gugelmin e Santos ${ }^{16} \mathrm{e}$ Leite et al. ${ }^{17}$, em investigações feitas entre povos indígenas, relataram maior frequência de excesso de peso entre as mulheres. Importante ressaltar

Tabela 2. Estado Nutricional: Guarani, de acordo com o sexo.

\begin{tabular}{|c|c|c|c|c|c|c|}
\hline & \multicolumn{2}{|c|}{ Total $(n=62)$} & \multicolumn{2}{|c|}{ Masculino $(n=38)$} & \multicolumn{2}{|c|}{ Feminino $(n=24)$} \\
\hline & f & (\%) & f & (\%) & f & $(\%)$ \\
\hline Baixo peso & 03 & $(4,8)$ & 02 & $(5,3)$ & 01 & $(4,2)$ \\
\hline Eutrófico & 37 & $(59,7)$ & 22 & $(57,9)$ & 15 & $(62,5)$ \\
\hline Sobrepeso & 20 & $(32,3)$ & 14 & $(36,8)$ & 06 & $(25,0)^{*}$ \\
\hline Obesidade & 02 & $(3,2)$ & 00 & $(0,0)$ & 02 & $(8,3)$ \\
\hline
\end{tabular}

"diferença significativa entre os sexos para $\mathrm{p}<0,05 . \mathrm{F}$ = frequência.

Tabela 3. Estado Nutricional dos Kaingang, de acordo com o sexo.

\begin{tabular}{|c|c|c|c|c|c|c|}
\hline & \multicolumn{2}{|c|}{ Total $(n=117)$} & \multicolumn{2}{|c|}{$\operatorname{Masculino}(n=80)$} & \multicolumn{2}{|c|}{ Feminino $(n=37$} \\
\hline & f & $(\%)$ & f & $(\%)$ & f & $(\%)$ \\
\hline Baixo peso & 01 & $(0,9)$ & 01 & $(1,25)$ & 00 & $(0,0)$ \\
\hline Eutrófico & 53 & $(45,3)$ & 41 & $(51,25)$ & 12 & $(32,4)$ \\
\hline Sobrepeso & 48 & $(41,0)$ & 31 & $(38,75)$ & 17 & $(45,9)$ \\
\hline Obesidade & 15 & $(12,8)$ & 07 & $(8,75)$ & 08 & $(21,7)^{*}$ \\
\hline
\end{tabular}

"diferença significativa entre os sexos para $\mathrm{p}<0,05$. $\mathrm{f}=$ frequência. que diferentes prevalências têm sido encontradas em estudos com diversas etnias. Um dos motivos pode ser o método para avaliação do estado nutricional, principalmente o IMC, que apesar de ser muito utilizado não permite diferenciar massa magra de gordura corporal. Além disso, outro fator que deve ser levado em conta é que no Brasil estudos com populações indígenas adotam os pontos de cortes utilizados para população em geral, dificultando ainda mais a avaliação de diferentes grupos étnicos ${ }^{18}$.

Nos últimos anos tem sido observado que os povos indígenas no Brasil estão expostos à transformações ambientais e socioeconômicas que os colocam em situação de alta vulnerabilidade frente a problemas de ordem alimentar e nutriciona ${ }^{19}$. Apesar da carência de estudos em nível nacional com esta população, algumas pesquisas isoladas em comunidades indígenas vêm mostrando uma mudança no perfil antropométrico e nutricional, caracterizada por uma diminuição de sujeitos com baixo peso ou peso normal e um aumento crescente e rápido de indivíduos com sobrepeso ou obesidade ${ }^{13,20,21}$.

Muitas pesquisas realizadas nos últimos dez anos, com foco na epidemiologia nutricional dos povos indígenas, mostram elevada frequência de sobrepeso e obesidade entre os adultos, em muitos casos superiores a $60 \%{ }^{12,13,15-17,20,22-25}$. Neste sentido, o cenário encontrado entre os adultos Kaingang e Guarani assemelha-se aos demais estudos realizados.

Para Haquim ${ }^{21}$, a explicação para este quadro está no fato de que nos últimos anos está havendo grandes mudanças socioculturais, econômicas e ambientais nas comunidades indígenas, consequentes de sua interação com a sociedade, levando à quebra de regras tradicionais, assalariamento, introdução errônea de alimentos industrializados, sedentarismo, escassez de terra para plantio, dentre outras. Diante disso, e em razão da elevada prevalência de sobrepeso e obesidade verificada em nosso estudo, pressupõe-se que esteja ocorrendo uma substituição dos hábitos alimentares tradicionais entre os Guarani e Kaingang do Paraná, com a incorporação de práticas comuns da população urbana, principalmente em relação aos alimentos industrializados.

De acordo com os resultados observados neste estudo, é importante salientar a necessidade emergente de acompanhamento e monitoramento da população indígena, avaliada por profissionais especializados, para identificar os problemas relacionados ao estilo de vida dessa população. Por exemplo, no tocante ao sedentarismo este 
problema pode ter sido agravado com a política de confinamento dessas populações, outrora nômades, em aldeias. Por isso, a introdução de exercícios físicos junto ao controle nutricional nas comunidades deve considerar os aspectos estruturais envolvidos.

\section{Conclusão}

Por meio deste estudo transversal, identificou-se um quadro preocupante no estado nutricional em indígenas Guarani e Kaingang avaliados, sendo observada elevada prevalência de sobrepeso e obesidade, principalmente entre o sexo feminino. Os resultados ressaltam a necessidade urgente de ações do poder público em comunidades indígenas, no sentido de orientar esta população a adquirir hábitos de vida mais saudáveis, prevenindo desta forma o surgimento de agravos à saúde causados pelo excesso de peso corporal. Além disso, os dados apontam para a necessidade emergente de uma abordagem mais intensa direcionada para o controle dos fatores de risco e na prevenção de doenças cardiovasculares nestas comunidades. Recomendações para o controle do peso corporal, o abandono do hábito de fumar, evitar consumo excessivo de sal e gorduras saturadas e a prática de atividades físicas com regularidade, são medidas essenciais na abordagem das doenças cardiovasculares. Nas políticas indígenas esses fatores devem vir integrados, já que os mesmos se manifestam interdependentes na promoção da saúde.

Estudos com outras etnias e amostras maiores são necessários, pois podem ser importantes preditores de saúde ao presente e ao futuro dos indígenas do Brasil.

O presente estudo não apresentou amostragem representativa das etnias estudadas. Tratase de amostra de conveniência de indígenas que participaram e/ou assistiram a um determinado evento. Sendo assim, sugere-se que outros estudos com amostras maiores e representativas sejam realizados a fim de determinar prevalências mais fidedignas.

\section{Colaboradores}

JD Boaretto, CAM Fernandes e GGA Pimentel participaram de todas as etapas do processo de elaboração do artigo.

\section{Agradecimentos}

Ao CNPq e à Rede CEDES pelo suporte financeiro. 


\section{Referências}

1. Instituto Brasileiro de Geografia e Estatística (IBGE). Censo Demográfico. Brasília: IBGE; 2014.

2. Instituto Socioambiental (ISA). Povos Indígenas no Brasil. São Paulo: ISA; 2014.

3. Hennerich JE. Aldeias Indígenas do Paraná. Olhares de Guarani para Guarani. Guarapuava: Unicentro; 2007.

4. Mota LT. As guerras dos índios Kaingang: a história épica dos indios Kaingang no Paraná (1769-1924). Maringá: Eduem; 2009.

5. Boaretto JD. Danças Indígenas Kaingang no Paraná: possibilidades de intervenção educacional [dissertação]. Maringá: Universidade Estadual de Maringá; 2012.

6. Mota LT, Assis VS. Populações indígenas no Brasil: histórias, culturas e relações interculturais. Maringá: Eduem; 2008.

7. Saad MBNL. Saúde e Nutrição Terena: Sobrepeso e Obesidade [dissertação]. Campo Grande: Universidade Federal de Mato Grosso do Sul; 2005.

8. Taddei JA, Lang RMF, Longo-Silva G, Toloni MHA. Nutrição em Saúde Pública. Editora Rubio; 2011.

9. Ribas DLB, Leite MS, Gugelmin AS. Perfil nutricional dos povos indígenas do Brasil. In: Barros DC, Silva DO, Gugelmin AS, organizadores. Vigilância alimentar e nutricional para saúde indígena. Rio de Janeiro: Fiocruz; 2007. p. 22-43.

10. Fundação Nacional do índio. O indio hoje. Brasília: Funai; 2014.

11. Brasil. Ministério da Saúde (MS). Conselho Nacional de Saúde. Resolução no 196 de 10 de outubro de 1996. Diretrizes e Normas Regulamentadoras de Pesquisas Envolvendo Seres Humanos. Diário Oficial da União 1996; 16 out.

12. Leite MS. Avaliação do Estado Nutricional da População Xavante de Sangradouro-Volta Grande, Mato Grosso [dissertação]. Rio de Janeiro: Fiocruz; 1998.

13. Capelli JCS, Koifman S. Avaliação do estado nutricional da comunidade indígena Parkatêjê, Bom Jesus do Tocantins, Pará, Brasil. Cad Saude Publica 2001; 17(2):433-437.

14. Tavares FG, Coimbra Junior CEA, Cardoso AM. Níveis tensionais de adultos indígenas Suruí, Rondônia, Brasil. Cien Saude Colet 2013; 18(5):1399-1409.

15. Salvo VLMA, Rodrigues D, Baruzzi RG, Pagliaro H, Gimeno SGA. Perfil metabólico e antropométrico dos Suyá. Parque Indígena do Xingu, Brasil Central. Rev Bras Epidemiol 2009; 12(3):458-468.

16. Gugelmin AS, Santos RV. Ecologia humana e antropometria nutricional de adultos Xavánte, Mato Grosso, Brasil. Cad Saude Publica 2007; 17(2):313-322.
17. Leite MS, Santos RV, Gugelmin AS, Coimbra Junior CE. Crescimento físico e perfil nutricional da população indígena Xavante de Sangradouro-Volta Grande, Mato Grosso, Brasil. Cad Saude Publica 2006; 22(2):265-227.

18. Simões BS, Machado-Coelho GLL, Pena, JL e Freitas SN. Perfil nutricional dos indígenas Xukuru-Kariri, Minas Gerais, de acordo com diferentes indicadores antropométricos e de composição corporal. Cien Saude Colet 2013; 18(2):405-411.

19. Coimbra JR., CEA, Santos RV, Escobar AL. Epidemiologia e saúde dos povos indígenas no Brasil. Rio de Janeiro: Editora Fiocruz, Abrasco; 2005.

20. Gimeno SGA, Rodrigues D, Pagliaro H, Cano EM, Lima EES, Baruzzi R. Perfil metabólico e antropométrico de índios Aruák: Mehináku, Waurá e Yawalapíti, Alto Xingu, Brasil Central, 2000/2002. Cad Saude Publica 2007; 23(8):1946-1954.

21. Haquim VM. Nutrição e alimentação dos povos indígenas: um desafio intercultural. CRN-3 Notícias 2008; 91:20-21.

22. Lourenço AE, Santos RV, Orellana JD, Coimbra Jr CEA. Nutrition transition in Amazonian: obesity and socioeconomic change in the Suruí Indians from Brazil. Am J Hum Biol 2008; 20(5):564-571.

23. Welch JR, Ferreira AA, Santos RV, Gugelmin AS, Werneck G, Coimbra Júnior CEA. Nutrition Transition, Socioeconomic Differentiation and Gender Among Adult Xavante Indians, Brazilian Amazon. Hum Ecology 2009; 37:13-26.

24. Associação Brasileira de Saúde Coletiva (Abrasco). I Inquérito Nacional de Saúde e Nutrição dos Povos Indígenas. Rio de Janeiro: Abrasco; 2009.

25. Castro TG, Schuch I, Conde WL, Veiga J, Leite MS, Dutra CLC, Zuchinali P. Estado Nutricional dos indígenas Kaingang matriculados em escolas indígenas do Estado do Rio Grande do Sul, Brasil. Cad Saude Publica 2010; 26(9):1766-1776.

Artigo apresentado em 21/09/2014

Aprovado em 13/03/2015

Versão final apresentada em 15/03/2015 